\title{
ASSOCIATIONS BETWEEN THE ANCIENT STAR CATALOGUES
}

\author{
DENNIS W. DUKE, Florida State University
}

There are just two substantial sources of star coordinates preserved for us from antiquity: the star catalogue of Ptolemy's Almagest 1 , and the rising, setting, and culmination phenomena, along with some star declinations and right ascensions, from Hipparchus' Commentary to Aratus ${ }^{2}$. Given the controversy associated with the idea that Ptolemy's catalogue is, in whole or in substantial part, a copy of an earlier but now lost catalogue of Hipparchus, it is of interest to try and establish clear and significant associations, or the lack thereof, between the two sets of ancient star data.

There have been two complementary efforts to clarify the possible associations. Vogt used the phenomena and declinations to reconstruct the ecliptical coordinates of some 122 stars in Hipparchus' Commentary that also appear in the Almagest catalogue. Vogt's conclusion was that since his reconstructed coordinates and the Almagest coordinates were, in general, different, Ptolemy did not obtain his data from Hipparchus. Vogt did notice five stars with very similar errors and concluded that Ptolemy probably did copy

those from Hipparchus. More recently, however, Grasshof $t^{4}$ has pointed out that there are several reasons to doubt Vogt's conclusion. Further, Grasshoff worked in the opposite direction, using the Almagest coordinates to compute the Hipparchan phenomena, and concluded, for two reasons, that the Almagest data and the Commentary data share a common origin. First, there are a number of stars that share large common errors, and it is highly unlikely that these agreements could be coincidental. Second, the correlation coefficients between the various error sets are typically large and statistically significant, and this also suggests a common origin of the two data sets. However, Grasshoff provided no analysis of the correlations to support this second conclusion.

In this paper I will

(1) analyze the correlations between the errors of the phenomena and the predictions of these phenomena following from the Almagest coordinates using extensive 
Monte Carlo simulations to show directly that it is highly improbable that the Almagest coordinates are substantially independent of the Commentary data;

(2) determine the systematic errors in the Hipparchan phenomena, and show that they are strongly related to the known systematic errors in the Almagest coordinates.

In both cases the comparisons point to a common origin of the Commentary data and the Almagest data.

\section{Analysis of the error correlations for the phenomena}

In the Commentary to Aratus, Hipparchus gave an extensive set of data on the phenomena relating specific points on the ecliptic to given stars. The phenomena are of five types. Type 1 is the degree of the ecliptic that rises simultaneously with a star. Type 2 is the degree of the ecliptic that culminates when a star rises. Type 3 is the degree of the ecliptic setting simultaneously with a star. Type 4 is the degree of the ecliptic that culminates as a star sets. Type 5 is the degree of the ecliptic that culminates when the star culminates. All five phenomena are easily calculated from the observer's geographical latitude and the star's coordinates and the obliquity of the ecliptic on the date of measurement (see Appendix A). For input to my calculations I have used the values of the phenomena found in Appendix C of Grasshoff's book. While there are some 619 phenomena listed in Grasshoff's table, the number of unique stars is just 292, compared to 1025 in the Almagest.

Let us denote by $\mathrm{H}$ the set of values of all the phenomena recorded by Hipparchus in part 2 of the Commentary. Then denote by $\mathrm{M}$ the values of the phenomena calculated using modern star coordinates precessed back to the epoch -128 (also listed in Grasshoff's table but recalculated by me). Finally, denote by A the values of the phenomena calculated using the star coordinates from the Almagest with $2^{\circ} 40^{\prime}$ subtracted from each longitude. Then the sets $\mathrm{H}-\mathrm{M}$ and $\mathrm{A}-\mathrm{M}$ represent the errors in the phenomena of Hipparchus and the predictions from the Almagest coordinates, respectively. 
Now among these sets of errors there are cases that are obviously anomalous. For example, for the star $\lambda$ Dra, Hipparchus quotes three values for $\Lambda_{5}: 119^{\circ}, 121^{\circ}$ and $151^{\circ}$. The value computed from modern data is about $120.7^{\circ}$, while the Almagest coordinates give about $119.2^{\circ}$. Thus the $151^{\circ}$ value is an obvious outlier, and should be omitted. Altogether, there are some 63 cases involving some 33 stars flagged in Grasshoff's Appendix $\mathrm{C}$ that are $\mathrm{5}^{5}$ 'cases of philological or astronomical uncertainty [and] are discussed by Manitius in extended footnotes...usually it indicates an obscure value...' Following Grasshoff, I have omitted these cases from further consideration. In addition, there are a few cases where a given value, e.g. $\Lambda_{5}=291.5^{\circ}$ for $\alpha \mathrm{Cyg}$, is simply duplicated in the tables. I have kept just one data point from each set of duplicates.

Let us now look at the correlations between the two sets of errors for each of the five types of phenomena. These correlations are shown in Figure 1. An analysis of figures similar to these led Grasshoff to conclude that the Almagest data and the Commentary data share a common origin ${ }^{6}$. First, there are a number of stars that share large common errors that are almost entirely statistical, and it is highly unlikely that these agreements are coincidental. Table 1 lists the top candidates for stars with large shared errors $\square$. Second, the overall correlation coefficients (see Table 2) between the various data sets are typically large and statistically significant, and this also suggested to Grasshoff a common origin of the data. However, Grasshoff did not publish the numerical values of the correlations and did not provide any analysis to support his conclusion.

Let us consider a model for these correlations that is based on the empirical observation that the variance of the Commentary errors is greater than the variance of the Almagestderived errors. This could be due to any number of reasons, but two of the most likely are (1) that the Commentary phenomena are rounded off to degrees or half-degrees, while the Almagest coordinates are generally given to $10^{\prime}$ precision (although the real accuracy after adjusting for outliers, is about 2-3 times larger than 10'), and (2) that there is no assurance that the Commentary phenomena are adjusted to the same epoch ${ }^{2}$, and this would add additional noise. 
Whatever the reasons, the empirical observation suggests the following model for the errors: the Almagest errors are $\varepsilon_{i}$, where $\varepsilon$ has mean zero and variance $\sigma_{A}^{2}$, while the Commentary errors are $\varepsilon_{i}+\eta_{i}$, and these have mean zero and variance $\sigma_{H}^{2}$, while $\varepsilon$ and $\eta$ are completely uncorrelated. The Commentary and Almagest errors share the $\varepsilon$ errors, but the added $\eta$ errors account for the fact that ${\sigma_{H}^{2}}^{2}>{\sigma_{A}}_{A}$. The most plausible source of the added $\eta$ errors is, of course, that Hipparchus used a globe to obtain his numbers for the phenomena. Under these assumptions, the correlation $r$ between the two sets of errors is just $r=\sigma_{A} / \sigma_{H}$, and the percentage of shared variance can have any value between zero and unity, even when, by the model assumption, every star has been copied (although, of course, not from the Commentary, but from a presumed earlier source). Since all three of the numbers $r, \sigma_{A}$, and $\sigma_{H}$ are measured from the data, the model has no adjustable parameters and is easy to check. The results are given in Table 2 . We see that overall the agreement with the data is within reasonable expectations for a zero-parameter model, and supports the idea that the Commentary data and the Almagest data share a common origin. Note also that this model provides an explicit counterexample to the assertions by Swerdlow 10 and Evans $\frac{11}{\text { that }} r$ is directly related to the percentage of copied coordinates. In fact, the claim that a low percentage of shared variance implies a large degree of independence of Ptolemy from the Commentary is purely an additional, model dependent assumption, completely independent of the numerical value of the correlations.

Now the reader might wonder whether the observed correlations are so strongly influenced by the pairs with large errors that, if these pairs were removed, the correlations would be much smaller and the evidence for association between the phenomena and the Almagest errors would weaken or vanish. Therefore, let us make the hypothesis that the Almagest errors are completely independent of the errors in the phenomena, and see to what level we can test that hypothesis. If we cannot reject that hypothesis, then we will not, of course, have proved that the Almagest is really independent. But if we can reject that hypothesis, then we will have proved that the Almagest cannot be independent, subject to whatever other assumptions are present in the model. 
Under the hypothesis, we assume that Ptolemy measured the star coordinates as he claimed in the Almagest, and so the data set that appears in the Almagest would be just the accurate positions of the stars at his epoch of +137 plus some measurement errors, and these errors are uncorrelated with the errors in the Commentary phenomena. Now in Ptolemy's case we know that the measurement errors are not totally random because it has been known for centuries 2 that Ptolemy's longitudes have an average systematic error of about $-1^{\circ}$. Further, Peters ${ }^{13}$ also pointed out that there are substantial periodic errors in Ptolemy's data in both longitude and latitude.

A method to estimate the systematic errors for each Almagest star is presented later in this paper (see section 3), but for now let us just assume that the systematic errors are known for each star, and therefore that a given Almagest coordinate is just the accurate value of that coordinate in +137 , plus the estimated systematic error in that coordinate, plus a random statistical error. This statistical error, $\varepsilon_{\lambda}$ or $\varepsilon_{\beta}$, will have mean zero and a standard deviation that varies somewhat across the sky. Using the actual Almagest coordinates, I have estimated the standard deviations of $\varepsilon_{\lambda}$ and $\varepsilon_{\beta}$ as 31.6' and 32.3', respectively, for stars in Ptolemy's northern constellations, $40.5^{\prime}$ and 30.1' for stars in the zodiacal constellations, and $44.6^{\prime}$ and $50.4^{\prime}$ for stars in the southern constellations. These numbers are estimated without eliminating any outliers $\frac{14}{4}$ in Ptolemy's data, and the resulting larger variances make it even more difficult to reject the hypothesis under consideration, that Ptolemy's errors are uncorrelated with the Commentary errors.

Under this assumption, then, the Almagest data are just one particular realization of a set of star measurements in +137 , subject to the assumed systematic and statistical errors, and we can also generate any number of other equally probable realizations by just substituting new sets of statistical errors with the variances mentioned above. Any one of these synthetic data sets will therefore look similar to the actual Almagest data, especially including the systematic errors.

Now if the hypothesis is true, then the correlations $r_{i}, i=1-5$, between the Commentary phenomena errors and the errors predicted using the actual Almagest data are entirely due 
to chance. Therefore, consider the set of correlations $\left\{r_{i}^{\prime}\right\}$ computed from the Commentary phenomena errors and the errors predicted using a large number (say 1,000) of the synthetic data sets. We can now test how probable it is that the actual Almagest data set is similar to the synthetic data sets by asking how often an observed $r_{i}^{\prime}$ exceeds the value of $r_{i}$. If the Almagest data are really similar to the synthetic data, then this will happen often, and on average about $50 \%$ of the time. If the Almagest data are not similar, then it will happen very infrequently, and we can use this to quantitatively reject with a known confidence level the hypothesis that the Almagest data were measured in +137 with known systematic errors and a set of random statistical errors with the variances observed in the Almagest data.

It is highly improbable that the hypothesis is completely true, of course, because of the cases of large common errors that we have already discussed. It is possible, though, that if we just exclude those cases, then the remaining stars were independently measured by Ptolemy ${ }^{15}$. By gradually throwing out the stars with large common errors, then, we can test this hypothesis as follows: consider a cutoff value for Hipparchus' errors, and let us exclude from further consideration any star that has an error that exceeds the cutoff (in absolute value, of course). We do this separately for each of the five phenomena and for a range of cutoff values from $6^{\circ}$ down to $1^{\circ}$, this latter limit being already significantly less than the observed standard deviation of the phenomena errors (see Table 2).

For the stars that survive the cutoff, we compute the correlations $r_{i}$ between the Commentary phenomena errors and the errors in the phenomena predicted from the Almagest. We then generate 1,000 synthetic data sets, and report in Table 3, for each value of the cutoff and for each of the five phenomena, the percentage of times that a synthetic $r_{i}^{\prime}$ exceeds the observed $r_{i}$. We see that even in the case of a cutoff as low as $1^{\circ}$, we can reject with greater than $98 \%$ confidence the hypothesis that the Almagest data were measured independently of the Commentary phenomena. This conclusion remains if the Almagest systematic errors are not used in the simulation. 
In the simulation just described, each of the synthetic data sets is composed entirely of synthetic values. If we relax this by putting $50 \%$ of the coordinate pairs equal to the Almagest values, then the $r_{i}{ }^{\prime}$ will tend to be larger, and the percentage of time that $r_{i}{ }^{\prime}$ exceeds the observed $r_{i}$ is about $4 \%, 1 \%, 7 \%, 6 \%$, and $7 \%$, respectively, for the five phenomena and for a cutoff of $1^{\circ}$. These values are right on the edge of the usual $95 \%$ limit for hypothesis rejection. If we increase the number of Almagest values in the synthetic data set to $80 \%$ (and keep the cutoff at $1^{\circ}$ ), then the five percentages are $13 \%$, $10 \%, 20 \%, 20 \%$, and $21 \%$ - too large for hypothesis rejection, but small enough to indicate that the amount of copying is probably quite large. Of course, this cutoff strategy already strongly favors the assumption of independent data in the Almagest, for there is no obvious reason to assume that if Ptolemy copied, he copied only the stars with the largest errors. The conclusion that the amount of copying was substantial appears to be inescapable.

I have further tested this entire procedure as follows. First, we replace the Almagest data with a new set of coordinates that is known to not be a copy of the underlying Commentary coordinates (by simply using for A one of the synthetic data sets). Then we compute the $r_{i}$ for this set of data, and we again generate 1,000 new synthetic data sets and ask how often the resulting $r_{i}{ }^{\prime}$ exceeds $r_{i}$. The results are as expected: the $r_{i}$ are small, and $r_{i}{ }^{\prime}$ exceeds $r_{i}$ about $50 \%$ of the time. This shows that if the Almagest data were really independent of the Commentary, the above analysis would have revealed that fact.

Next, I have constructed a synthetic set of Commentary phenomena that are known to be based on the Almagest coordinates (by simply adding gaussian noise to the phenomena computed using the Almagest coordinates). Then I have computed the $r_{i}$ for this set of data, and again generated 1,000 new synthetic data sets and ask how often the resulting $r_{i}{ }^{\prime}$ exceeds $r_{i}$. The results are again as expected: the $r_{i}$ are fairly large, and the probability that $r_{i}{ }^{\prime}$ exceeds $r_{i}$ is very low, even for a cutoff as low as $1^{\circ}$. This shows that the analysis above does reliably reject the proposed independence when it is not present. 
In addition to correlations, the analysis has also been performed using $\chi^{2}$ as the test statistic. Indeed, let us define $\chi^{2}=\sum_{i}\left(H_{i}-A_{i}\right)^{2}$, where it is understood that we have a separate $\chi^{2}$ for each of the five types of phenomena, and the sum runs over the data points for that phenomenon. As before we vary the cutoff from 6.0 down to 1.0, generate 1,000 synthetic data sets for each cutoff, and this time we ask how frequently the value of $\chi^{2}$ for the synthetic data set is less than the $\chi^{2}$ comparison of the actual Commentary and Almagest data. The result is that $\chi^{2}$ is smaller less than $1 \%$ of the time, even for a cutoff of $1^{\circ}$, for each of the five phenomena. Thus we see that, point-by-point, the actual Almagest data predict the Commentary data much better that expected if the Almagest data were really measured independently of the Commentary data. And as before, we can reject at about the $99 \%$ confidence level the hypothesis that the Almagest data are independent of the Commentary data.

This analysis is strictly relevant, of course, only for those stars that are common to the Commentary and the Almagest. As mentioned above, the number of such stars is just 292, compared to 1025 in the Almagest. On the other hand, the Commentary stars are distributed fairly uniformly on the celestial sphere (122 in the north, 97 in the zodiac, and 73 in the south), so there is no reason to suppose that while Ptolemy did copy these stars, he measured independently all the others not in the Commentary. In any event, no analysis can ever prove that the Almagest data are completely copied from a lost Hipparchan data set, for it is always possible that Ptolemy, or even some later scholar, edited or supplemented the data to a very minor degree that would be impossible to detect. Probably the fairest conclusion from the present analysis is that it is highly probable that Ptolemy did copy substantial parts of his catalogue from prior sources closely associated with the Commentary phenomena, in contradiction to what he wrote in the Almagest text, and far in excess of the $5-10 \%$ previously proposed by Vogt and Swerdlow, or even the 21\% upper limit suggested by Evans. 


\section{Comparison of the systematic errors in the Commentary and Almagest data}

We have seen in the previous sections that the total errors, which are dominantly statistical, force us to reject the hypothesis that the Almagest errors are independent of the Commentary errors. We shall see in this section that the systematic errors in the two ancient data sets also lead us to the same conclusion.

Let us begin by reviewing the method suggested by Dambis and Efremov ${ }^{16}$ for estimating the systematic errors in the Almagest star catalogue. Considering first the latitudes $\beta$, Dambis and Efremov suppose that

$$
\Delta \beta=\beta_{A}-\beta_{c}(T)=\Delta \beta_{s y s}+\varepsilon,
$$

where $\beta_{A}$ is the value recorded in the Almagest star catalog, $\beta_{c}(T)$ is the value computed for some epoch $T$ using modern star coordinates and precession theory, $\Delta \beta_{\text {sys }}$ is the systematic error in the ancient catalogue value, and $\varepsilon$ is the random statistical measurement error in the ancient catalogue value and is assumed to have mean zero and variance $\sigma^{2}$. In order to isolate the systematic error $\Delta \beta_{\text {sys }}$, we consider each Almagest star in turn, and we then consider a number $n$ of nearest neighbor stars of that target star (typically $n$ is 16 in this paper). We average $\Delta \beta$ over these stars, obtaining

$$
<\Delta \beta>_{n}=\Delta \beta_{\text {sys }}+\varepsilon^{\prime}
$$

where the random variable $\varepsilon^{\prime}$ has mean zero and variance $\sigma^{2} / n$ due to the averaging. In fact, Dambis and Efremov compute the median of the $n$ values rather than the mean, since the median is somewhat more robust but still has the $1 / n$ effect in reducing the variance. I have checked it makes no significant difference in the final conclusions which choice is made, and in this paper I have used averaging. Note that we assume that the systematic error term is the same for both the target star and each of the nearest neighbor stars, due to their mutual locality on the celestial sphere. The important point is that the 
variance of the noise term $\varepsilon^{\prime}$ is reduced by a factor $1 / n$, and so $\left\langle\Delta \beta>_{n}\right.$ is a good estimate of the systematic error $\Delta \beta_{s y s}$ in the measurement of the target star.

The entire procedure outlined above is also performed for the ecliptic longitudes $\lambda$, with the proviso that we always use the quantity $\lambda \cos \beta$ in order to appropriately weight the errors in $\lambda$ that naturally increase with latitude.

Next we estimate the systematic errors in the phenomena measurements. Because the phenomena are, in general, only quoted to a precision of a $1^{\circ}$ or a $12^{\circ}$, the amount of noise in the data is somewhat larger than in the Almagest data. In addition, the density of stars is considerably less than in the Almagest catalogue, and so it is necessary to use a different method to estimate the systematic errors in the Commentary data. Therefore I have used a standard smoothing algorithm $\frac{18}{18}$ LOWESS that makes no assumption about the form of the systematic errors and is robust against outliers. However, to be safe I have also eliminated outliers using a standard algorithm 19 that is generally preferable to the familiar $3 \sigma$ or $4 \sigma$ cutoff method. The uncertainties in these systematic error determinations have been estimated with a Monte Carlo simulation. Finally, I have checked that alternative smoothing methods such as cubic-spline interpolation give very similar results to the LOWESS algorithm.

As above, denote by $\mathrm{H}$ the values of the phenomena recorded by Hipparchus in the Commentary, denote by $\mathrm{M}$ the values of the phenomena calculated using modern star coordinates precessed back to the epoch -128 , and denote by A the values of the phenomena calculated using the star coordinates from the Almagest with $2^{\circ} 40^{\prime}$ subtracted from each longitude. Finally, denote by $\mathrm{A}^{\prime}$ the values of the phenomena calculated using the coordinates from the Almagest but with $3^{\circ} 40^{\prime}$ subtracted from each longitude, and also the estimated systematic errors $\langle\Delta \beta\rangle_{n}$ and $\langle\Delta \lambda\rangle_{n}$ subtracted from each latitude or longitude, respectively. Then we look at the quantities $\mathrm{H}-\mathrm{M}, \mathrm{A}-\mathrm{M}$, and $\mathrm{A}^{\prime}-\mathrm{M}$ as a function of the longitude of each of the stars, and use the smoothing algorithm to estimate the systematic errors in each set. The results for the estimates of the systematic errors in the five different types of phenomena and the three different data sets are shown in 
Figure 2, and a sample of the 1- $\sigma$ uncertainties are indicated by the errors bars in the figures.

First, note that the systematic errors for the set $A^{\prime}-M$ are overall relatively small and nearer zero than for the other sets. This is just what one expects since the coordinates in the set $\mathrm{A}^{\prime}$ are corrected for systematic error and hence should agree, apart from residual noise, with the results for the set $\mathrm{M}$, which are, of course, free of errors. The systematic errors for $\mathrm{A}^{\prime}-\mathrm{M}$ appear to have a sinusoidal nature, but $\mathrm{I}$ have been unable to discover its source. I have verified that it is not due to a mis-set of the obliquity or a mis-set in the observer's geographical latitude.

Note further, though, that the errors for the sets $\mathrm{H}-\mathrm{M}$ and $\mathrm{A}-\mathrm{M}$ tend to track each other fairly closely, well within the bounds of statistical variation estimated by Monte Carlo, and are generally quite distinct from the $\mathrm{A}^{\prime}-\mathrm{M}$ points, indicating the presence of a common pattern of systematic errors in the two data sets, which must result from the (roughly) periodic errors in the Almagest data. In addition to the shape variation, the quantities $\mathrm{H}-\mathrm{M}$ and $\mathrm{A}-\mathrm{M}$ are systematically low, and that might suggest that they correspond to an epoch earlier than -128 . By determining the dates for which the average value of the systematic error is zero, I estimate the average epoch for types 1-5 at about $164,-159,-131,-131$, and -145 , respectively, with uncertainties of at least $\pm 10-15$ years. However, there are other ways to account for the relatively small systematic level shift in the errors, one of the simplest being that if the $1^{\circ}$ or $1 / 2^{\circ}$ precision in the Commentary data indicates rounding down, as apparently was the general custom, then that alone would bias the apparent dates lower by about 35 years or so. Thus, those dates should probably not be considered at all conclusive, and probably not even significant.

We see that the comparisons of the systematic error estimates for the phenomena using both Commentary and Almagest data do not agree with each other exactly, but neither should we expect exact agreement, given the amount of statistical processing required to estimate the systematic errors. Nevertheless, it is clear that the agreement is close enough that the most reasonable conclusion is that both sets of data share the same, or closely 
related, systematic errors. Still, some readers will perhaps wonder, in spite of the evidence presented in section 1 above, whether it is possible that the Commentary and Almagest data were measured independently, some 265 years or more apart, and that both sets of data would have the same, or nearly the same, pattern of systematic errors.

First, let us remember that we have not shown that both sets of data have the same systematic errors in their own epoch. Rather, we have shown that if the Almagest data is simply transformed to epoch -128 by subtracting $2^{\circ} 40^{\prime}$ from the longitudes, then the systematic errors are very similar to the systematic errors in the Commentary data, both in shape, which is a reflection of the periodic terms, and in level, which is possibly, but not certainly, a reflection of a common mis-set of epoch relative to -128 , the conventionally accepted epoch for Hipparchus. Now, for one part of the systematic errors, this agreement is not only possible but also likely, even if the data were measured independently. This follows if we accept Ptolemy's claim ${ }^{20}$ that both he and Hipparchus used the same erroneous value for the obliquity of the ecliptic, and if we further assume that both observers mis-set their armillary spheres according to that error. It turns out, though, that predictions for the phenomena vary only weakly with variations in the obliquity, and the level of agreement displayed in Figure 2 does not change appreciably as $\varepsilon$ varies between $23^{\circ} 40^{\prime}$ and $24^{\circ}$. Therefore the common error in the obliquity is definitely not the source of agreement in the phenomena systematic error comparisons.

Second, the next obvious source of common systematic error is due to the common solar theory ${ }_{2}$ of Hipparchus and Ptolemy. However, in order to independently incur similar systematic errors due to the solar theory, both would have had to follow very similar measuring protocols ${ }^{22}$, down to measuring the same stars at the same time of year, at the same time of night, with the same elongation of the moon from the sun, with respect to the same reference stars, etc. When considering this source of error, it is crucial to keep in mind the insight of Rawlins ${ }^{23}$ that the periodic errors in longitude and latitude are due to the interaction between the armillary instrument and the solar errors $\frac{24}{\text { Thus, the large }}$ longitudinal mis-set of the instrument that Ptolemy would have had to make implies a pattern of periodic errors, especially for the latitudes, that is simply missing from the 
data. Therefore, in order for Ptolemy to incur just those systematic errors that are seen in the Almagest data assuming a true epoch of +137 , he would have needed a very particular set of additional errors, perhaps instrumental, which when combined with the unavoidable and large errors from the solar theory, produce just the pattern of errors needed to agree with the errors that Hipparchus (or some observer) made 265 years earlier. Such a coincidence must be unlikely in the extreme.

Finally, if the errors for each observer are dominantly or even partly instrumental ${ }^{25}$, then it is extremely unlikely that two instruments built 265 years apart with undoubtedly varying degrees of precision would share the same instrumental errors (other than the obliquity mis-set).

Therefore I believe we can safely discard any idea that the agreement between the various sets of systematic errors would result from shared errors in the obliquity or the solar theory. Indeed, given the broad range of opportunities for systematic errors to occur, it is perhaps a larger mystery how relatively simple periodic patterns survive in the data at all.

\section{Summary}

We have seen that comparing both the systematic errors and the statistical errors leads to the same conclusion: the Commentary and Almagest data share a common origin of some sort. Probably the most plausible scenario is that Hipparchus had a now lost star catalogue. Exactly how and when the Commentary data might be related to that catalogue is, to say the least, not clear. Most previous analyses 26 of the Commentary data suggest an

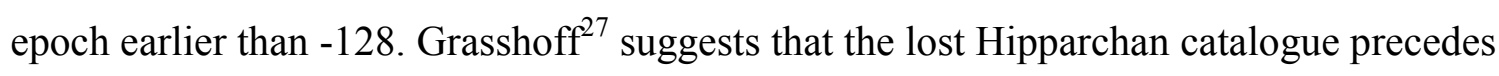
the Commentary, and that Hipparchus used a globe to estimate the phenomena quoted in the Commentary. Nadal and Brunet ${ }^{28}$ have examined the Commentary data in detail and reach a similar conclusion. Pickering 29 has pointed out that for three stars it appears that Hipparchus made a slip when computing the phenomena, and this scenario suggests that Hipparchus had a greater command of spherical trigonometry than is generally believed

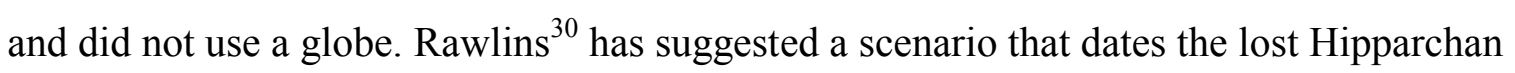


catalogue to -128 and accounts for the small negative error in the coordinates as a result of the time spent by the observer rotating and sighting the rings of the astrolabe. The idea that Hipparchus, sometime near to -128 and after he wrote the Commentary, for some

reason updated or measured again all his star data was dismissed by Neugebauer ${ }_{\text {as }}$ 'absurd', but I am aware of no facts which bear on such a position. Apparently it must be left to future work to further clarify the situation.

\section{Appendix A}

The problem is to compute the values of the five phenomena $\Lambda_{1}$ through $\Lambda_{5}$ given a pair of star coordinates, the obliquity of the ecliptic, and the observer's geographical latitude. If the given coordinates are ecliptical, convert them to equatorial using standard formula $\mathrm{e}^{22}$. Thus we have the right ascension $\alpha$, the declination $\delta$, the obliquity $\varepsilon$, and the geographical latitude $\varphi$. For types 1 and 2, compute the local sidereal time using $\theta=2 \pi-H+\alpha$, where the local hour angle $H$ is obtained from $\cos H=-\tan \delta \tan \varphi$. For types 3 and 4 use instead $\theta=H+\alpha$, while for type 5 use simply $\theta=\alpha$. Then for type 1 use

$$
\tan \Lambda_{1}=\frac{\cos \theta}{-\sin \varepsilon \tan \varphi-\cos \varepsilon \sin \theta}
$$

to carefully compute the inverse tangent (keeping the signs as shown, and probably using the $\operatorname{atan} 2(y, x)$ function in your programming language library, in order to get the correct quadrant for the angle). For type 2 the corresponding expression is

$$
\tan \Lambda_{2}=\frac{\sin \theta}{\cos \varepsilon \cos \theta}
$$

For type 3 use

$$
\tan \Lambda_{3}=\frac{-\cos \theta}{\sin \varepsilon \tan \varphi+\cos \varepsilon \sin \theta}
$$

while for type 4 use

$$
\tan \Lambda_{4}=\frac{\sin \theta}{\cos \varepsilon \cos \theta}
$$

i.e. the same as for type 2. Finally, for type 5 use

$$
\tan \Lambda_{5}=\frac{\sin \theta}{\cos \varepsilon \cos \theta}
$$


Note that while the formula for $\tan \Lambda$ is the same for types 2,4 , and 5 , the value of $\theta$ varies as mentioned above.

Pickering's analysis ${ }^{34}$ of the type 5 phenomena has uncovered three cases where Hipparchus' errors are consistent with a computational slip, wherein Hipparchus mistakenly used $\theta=\lambda$ instead of $\theta=\alpha$ as input to the equation for $\Lambda_{5}$.

\section{Acknowledgement}

I thank Paul Beaumont and Dennis Ridley for very helpful discussions about statistics. 
Table 1. Stars with large (usually $>1^{\circ}$ ) shared errors

\begin{tabular}{|c|c|c|c|c|}
\hline $\begin{array}{c}\text { Bailey } \\
\text { number }\end{array}$ & name & $\begin{array}{c}\text { type of } \\
\text { phenomenon }\end{array}$ & $\begin{array}{c}\text { Commentary } \\
\text { error }\end{array}$ & $\begin{array}{c}\text { Almagest } \\
\text { error }\end{array}$ \\
\hline 24 & $\alpha$ Uma & 5 & -1.7 & -1.4 \\
\hline 96 & v Boo & 4 & 0.9 & 1.2 \\
\hline 119 & $\alpha \mathrm{Her}$ & 5 & -2.1 & -1.2 \\
\hline 143 & т Her & 2 & -1.0 & -1.1 \\
\hline 167 & к Cyg & 2 & -2.2 & -0.6 \\
\hline 178 & $\zeta$ Cas & 1 & -1.7 & -1.8 \\
\hline 178 & & 2 & -1.7 & -1.8 \\
\hline 178 & & 5 & -1.0 & -1.1 \\
\hline 191 & N869 & 1 & -1.6 & -1.1 \\
\hline 285 & $\beta$ Sge & 2 & -1.5 & -1.1 \\
\hline 426 & $\theta \mathrm{Gem}$ & 1 & 4.1 & 4.1 \\
\hline 426 & & 2 & 3.0 & 3.5 \\
\hline 426 & & 5 & 3.1 & 2.8 \\
\hline 455 & I Cnc & 1 & -5.7 & -3.0 \\
\hline 455 & & 2 & -3.2 & -3.4 \\
\hline 455 & & 3 & 1.5 & 0.9 \\
\hline 455 & & 4 & 1.5 & 0.9 \\
\hline 498 & $\xi$ Vir & 2 & -1.3 & 1.0 \\
\hline 501 & $\beta$ Vir & 3 & -2.1 & -1.5 \\
\hline 501 & & 4 & -1.0 & -1.0 \\
\hline 509 & $\varepsilon$ Vir & 5 & -1.6 & -1.5 \\
\hline 592 & $\beta$ Sgr & 3 & -7.3 & -5.4 \\
\hline 592 & & 4 & -4.9 & -3.6 \\
\hline 646 & $\delta$ Aqr & 1 & -1.1 & -1.3 \\
\hline 707 & $\psi$ Psc & 5 & 1.8 & 3.0 \\
\hline 719 & $\rho$ Cet & 1 & -1.5 & -1.3 \\
\hline 805 & $\theta$ Eri & 1 & -2.5 & -2.7 \\
\hline 805 & & 2 & -2.9 & -3.4 \\
\hline 805 & & 3 & 5.8 & 6.8 \\
\hline 805 & & 4 & 6.8 & 8.0 \\
\hline 813 & $\beta$ Lep & 5 & 1.3 & 1.6 \\
\hline 892 & a Car & 3 & 5.1 & 4.2 \\
\hline 892 & & 4 & 5.0 & 4.7 \\
\hline 918 & п Нуа & 1 & 3.5 & 3.1 \\
\hline 918 & & 2 & 3.7 & 3.5 \\
\hline 918 & & 3 & -6.5 & -7.4 \\
\hline 918 & & 4 & -3.8 & -4.4 \\
\hline 969 & a Cen & 1 & 4.7 & 4.8 \\
\hline 969 & & 2 & 6.8 & 6.4 \\
\hline 992 & $\theta$ Ara & 1 & -1.6 & -3.0 \\
\hline 992 & & 2 & -2.5 & -3.7 \\
\hline 993 & a Ara & 5 & -1.4 & -2.2 \\
\hline
\end{tabular}




\begin{tabular}{|l|l|l|l|l|}
\hline & $\sigma_{\mathrm{A}}$ & $\sigma_{\mathrm{H}}$ & $\sigma_{\mathrm{A}} / \sigma_{\mathrm{H}}$ & $\mathrm{r}$ \\
\hline$\Lambda_{1}$ & 1.19 & 1.48 & 0.80 & 0.80 \\
$\Lambda_{2}$ & 1.34 & 1.55 & 0.87 & 0.81 \\
$\Lambda_{3}$ & 1.54 & 2.06 & 0.75 & 0.71 \\
$\Lambda_{4}$ & 1.49 & 1.76 & 0.85 & 0.69 \\
$\Lambda_{5}$ & 0.68 & 1.04 & 0.65 & 0.25 \\
$\Lambda_{\text {all }}$ & 1.16 & 1.49 & 0.78 & 0.66 \\
\hline
\end{tabular}

Table 2. The first column gives the type of variable in the correlation. The second column gives the standard deviation of the variable using the Almagest catalogue data as input. The third column gives the standard deviation of the variable using the Commentary data as input. The fourth and fifth columns should be equal if the simple model discussed in the text is relevant. The fact that they are generally close shows that the model in the text agrees reasonably well with the data.

\begin{tabular}{|c|c|c|c|c|c|}
\hline $\begin{array}{c}\text { cutoff } \\
\text { (degrees) }\end{array}$ & $\Lambda_{1}$ & $\Lambda_{2}$ & $\Lambda_{3}$ & $\Lambda_{4}$ & $\Lambda_{5}$ \\
\hline 6.0 & $0.0 \%$ & $0.0 \%$ & $0.0 \%$ & $0.0 \%$ & $0.6 \%$ \\
5.5 & $0.0 \%$ & $0.0 \%$ & $0.3 \%$ & $0.0 \%$ & $0.5 \%$ \\
5.0 & $0.0 \%$ & $0.0 \%$ & $4.1 \%$ & $0.0 \%$ & $0.9 \%$ \\
4.5 & $0.0 \%$ & $0.0 \%$ & $5.1 \%$ & $0.1 \%$ & $0.3 \%$ \\
4.0 & $0.0 \%$ & $0.0 \%$ & $4.3 \%$ & $0.0 \%$ & $0.2 \%$ \\
3.5 & $0.0 \%$ & $0.0 \%$ & $3.2 \%$ & $6.4 \%$ & $0.5 \%$ \\
3.0 & $0.0 \%$ & $0.0 \%$ & $5.8 \%$ & $6.0 \%$ & $3.3 \%$ \\
2.5 & $0.0 \%$ & $0.0 \%$ & $1.5 \%$ & $1.9 \%$ & $1.6 \%$ \\
2.0 & $0.0 \%$ & $0.1 \%$ & $0.6 \%$ & $0.1 \%$ & $3.4 \%$ \\
1.5 & $0.7 \%$ & $0.2 \%$ & $6.0 \%$ & $0.2 \%$ & $0.0 \%$ \\
1.0 & $0.4 \%$ & $0.2 \%$ & $1.6 \%$ & $1.5 \%$ & $1.6 \%$ \\
\hline
\end{tabular}

Table 3. The percentage of times (out of 1,000 tries) that the correlation of the synthetic data set with the Commentary data exceeds the correlation of the Almagest data with the Commentary phenomena. For a given value of the cutoff (first column), only errors less than that cutoff (in absolute value) are used to compute the correlation. 

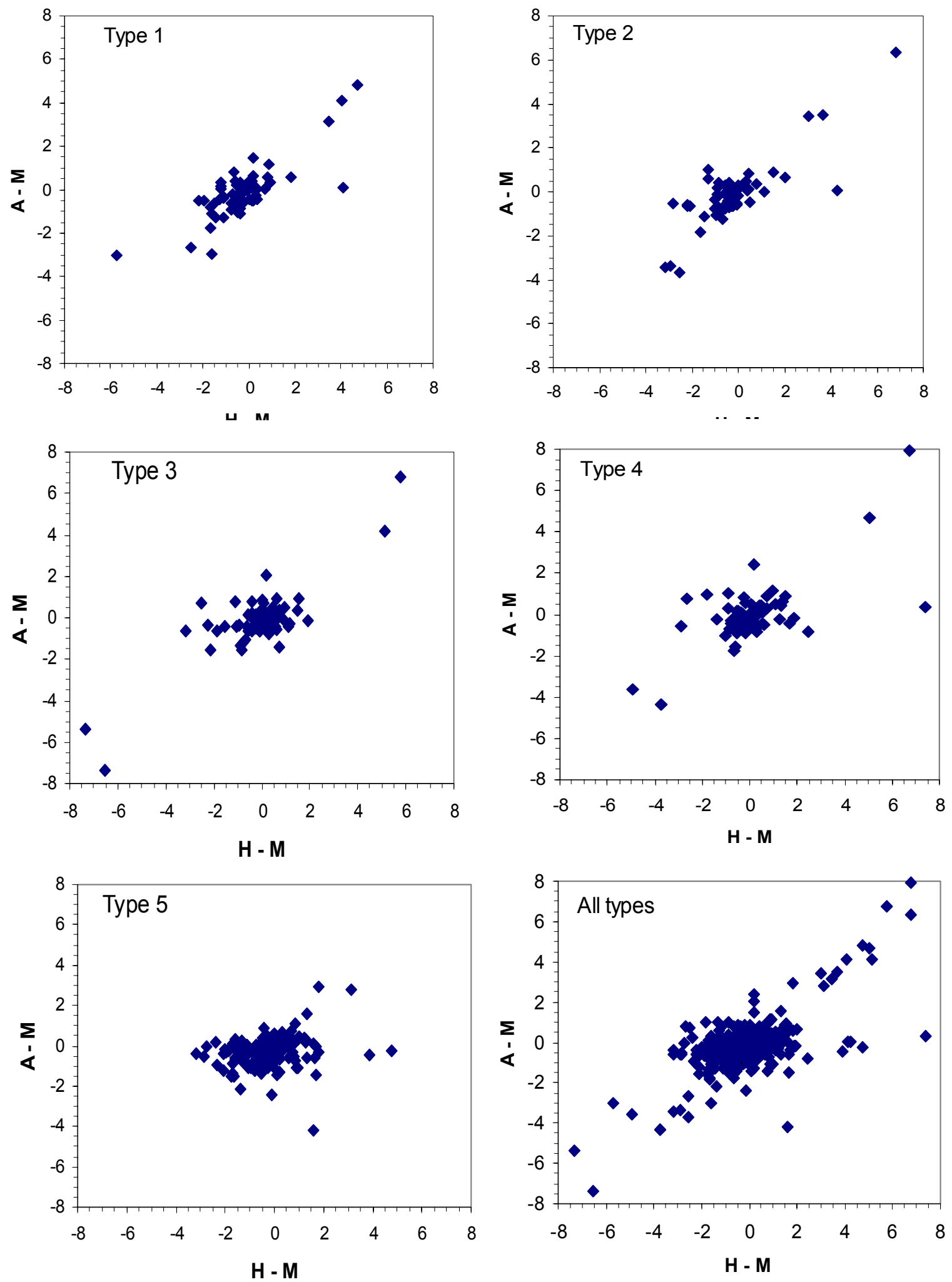

Figure 1. Correlations between the Commentary errors (x-axis) and the Almagest errors (y-axis). The units are degrees. 

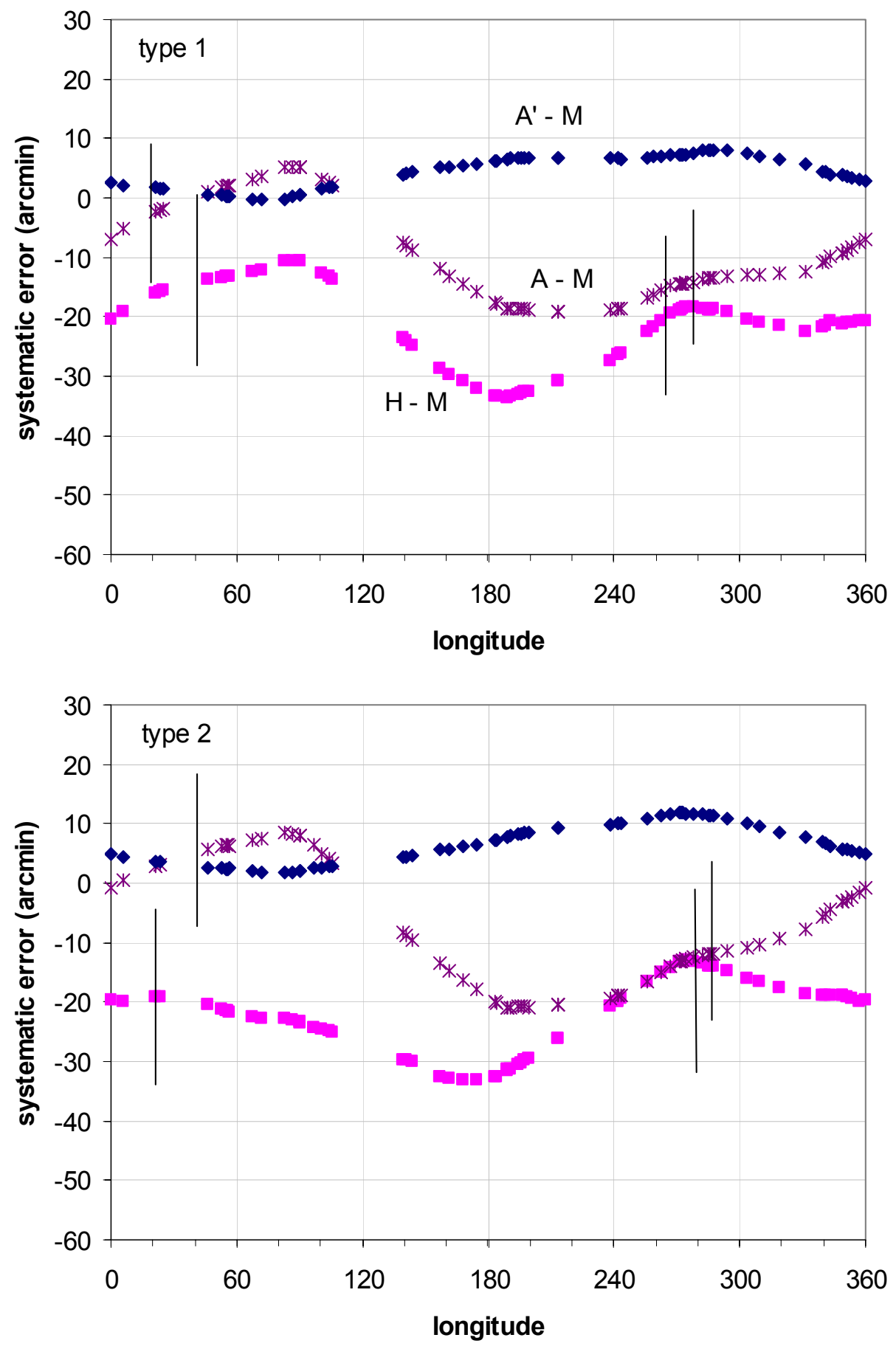

Figure 2. The systematic errors corresponding to the cases $\mathrm{H}-\mathrm{M}$ (boxes), $\mathrm{A}-\mathrm{M}$ (crosses), and $\mathrm{A}^{\prime}-\mathrm{M}$ (diamonds). The error bars shown are estimated by Monte Carlo and are about the same for each point, but only a few are shown in order to reduce visual clutter. 

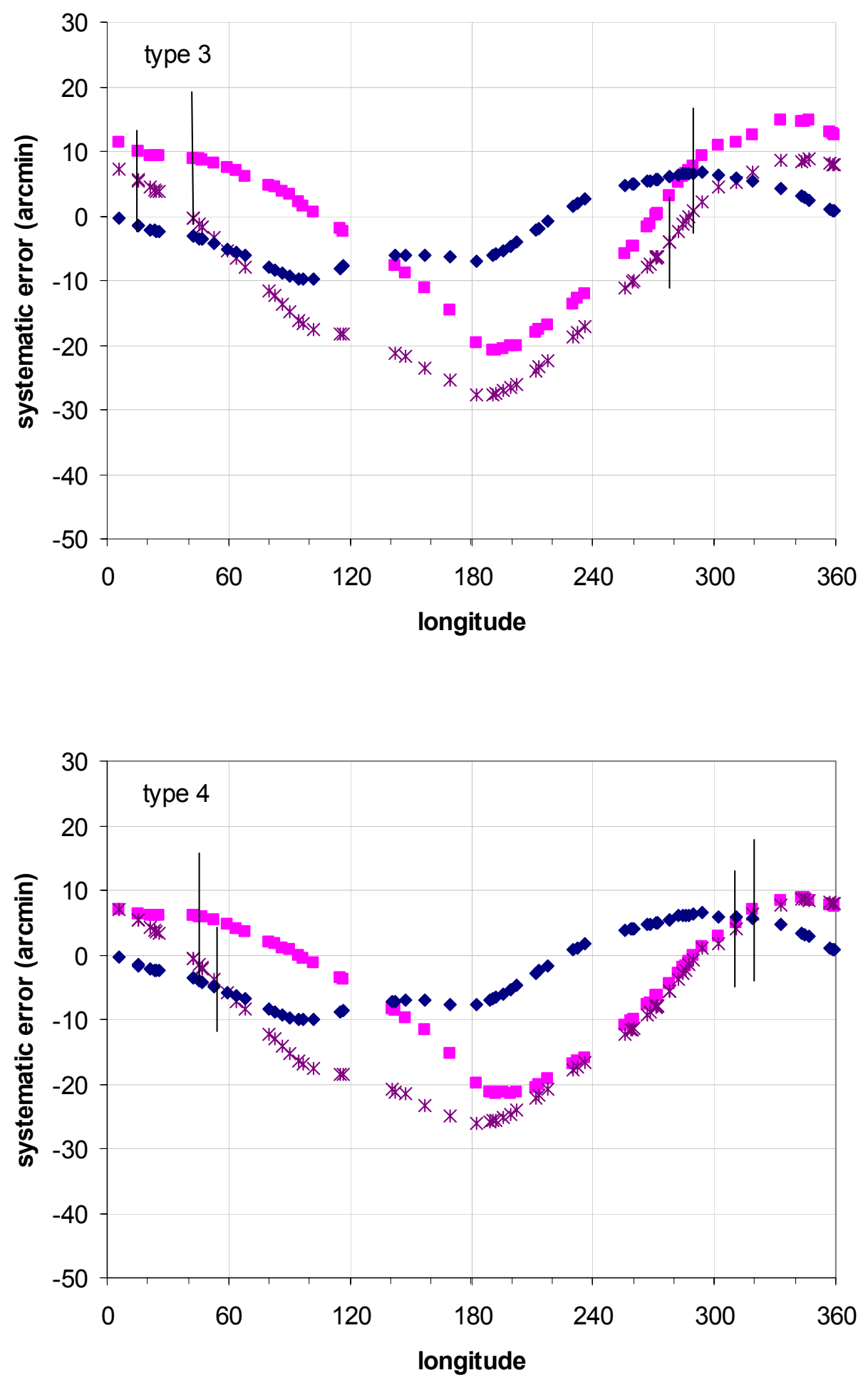

Figure 2 (continued) 


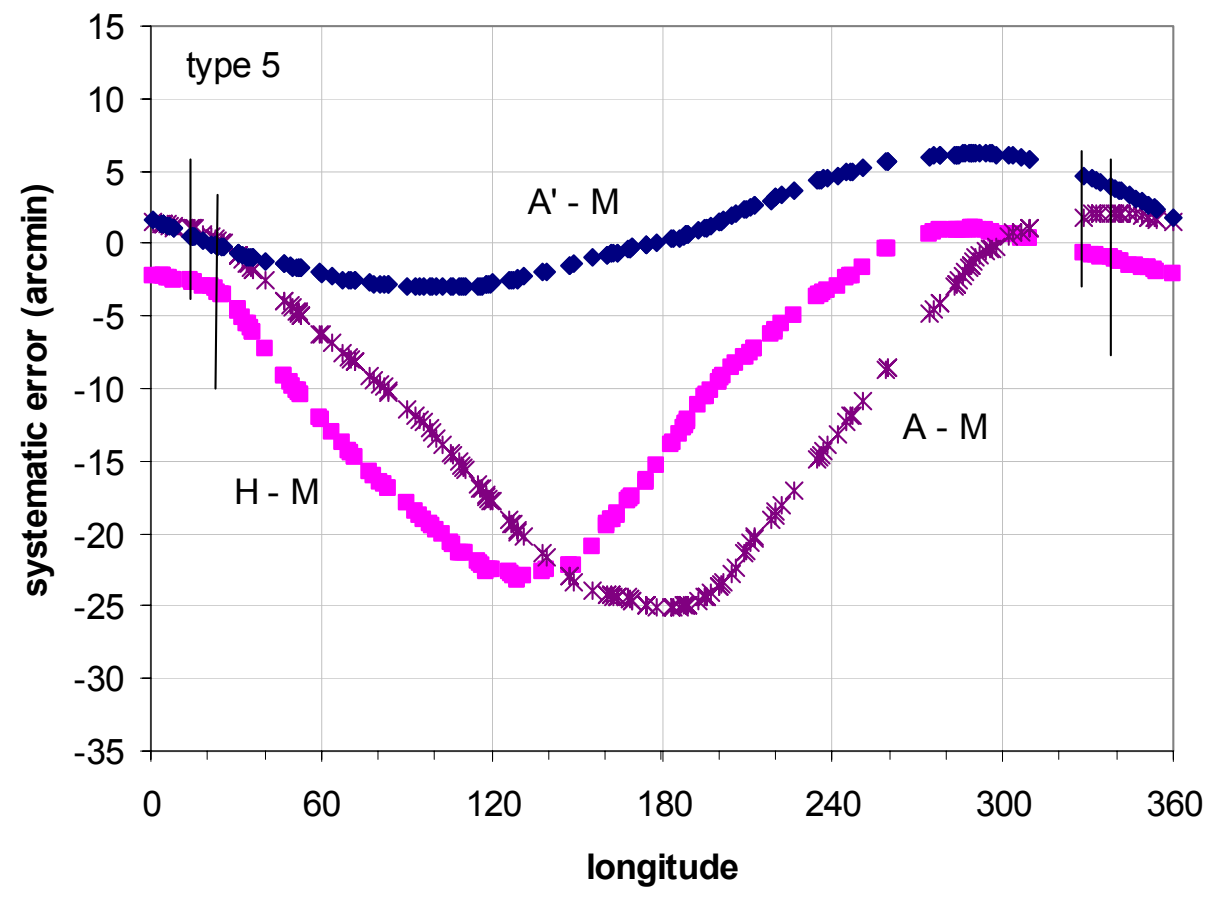

Figure 2 (continued) 


\section{REFERENCES}

${ }^{1}$ Ptolemy's Almagest, transl. by G. J. Toomer (London, 1984).

${ }^{2}$ Hipparchus, In Arati et Eudoxi phaenomena commentariorium, ed. and transl. by K. Manitius (Leipzig, 1894).

${ }^{3}$ H. Vogt, "Versuch einer Wiederstellung von Hipparchs Fixsternverzeichnis", Astronomische Nachtrichten, ccxxiv (1925), cols 2-54.

${ }^{4}$ G. Grasshoff, The history of Ptolemy's star catalogue (New York, 1990), 99-121.

${ }^{5}$ G. Grasshoff, op. cit. (ref. 4), 318.

${ }^{6}$ ibid., 178-92.

${ }^{7}$ Membership in Table 1 is not meant to necessarily imply that the coordinates were copied in the cases of the smaller errors.

${ }^{8}$ D. Duke, "Dating the almagest star catalogue using proper motions: a reconsideration", Journal for the history of astronomy, xxxiii(2002).

${ }^{9}$ This possibility must be allowed if Hipparchus wrote the Commentary before he discovered the effect of precession on star positions.

${ }^{10}$ N. M. Swerdlow, “The enigma of Ptolemy's catalogue of stars", Journal for the history of astronomy, xxiii (1992), 173-183.

11 J. Evans, "The Ptolemaic star catalogue", Journal for the history of astronomy, xxiii(1992), 64-68.

12 Tychonis Brahe Dani Opera Omnia, ed. J. L. E. Dreyer (Copenhagen, 1913-29), vol. II, p. 151.

${ }^{13}$ C. H. F. Peters, "Ueber die fehler des Ptolemaischen sternverzeichenisses", Vierteljahrsschrift Ast. Gesell (1877). The most accessible source of Peters' results is C. H. Peters and E. B. Knobel, Ptolemy's catalogue of stars: A revision of the Almagest (Washington, 1915).

${ }^{14}$ Estimates including a careful treatment of outliers are in ref. 8.

${ }^{15}$ Indeed, this is precisely the claim of Vogt (ref. 3), Swerdlow (ref. 10), and Evans (ref. 11). 
${ }^{16}$ A. K. Dambis and Yu. N. Efremov, "Dating Ptolemy's star catalogue through proper motions: the Hipparchan epoch", Journal for the history of astronomy, xxxi (2000), 115134.

${ }^{17}$ My data sources are given in ref. 8.

${ }^{18}$ W. S. Cleveland, Robust locally weighted regression and smoothing scatterplots, Journal of the American statistical association ,74 (1979), 829-36.

${ }^{19}$ F. E. Grubbs, "Procedures for detecting outlying observations in samples", Technometrics, 11 (1969), 1-21.

${ }^{20}$ Almagest, op. cit. (ref. 1), I.12, p. 63.

${ }^{21}$ Almagest, op. cit. (ref. 1), III.4, p. 153ff.

${ }^{22}$ Almagest, op. cit. (ref. 1), VII.4, 339-40. For a nice discussion of the measuring protocols, see J. Evans, "On the origins of the Ptolemaic star catalogue", Journal for the history of astronomy, xviii (1987), 233-39.

${ }^{23}$ D. Rawlins, "An investigation of the ancient star catalog”, Publications of the Astronomical Society of the Pacific, xciv (1982), 359-73.

${ }^{24}$ A casual reader of Grasshoff, op. cit. (ref. 4), 148-155 might lead one to think that the periodic errors can be identified directly with the solar errors, as in Grasshoff's Figures 5.19 and 5.20.

${ }^{25}$ A sample of possible instrumental errors is give in J. Evans, op. cit. (ref 33).

${ }^{26}$ H. Vogt, op. cit. (ref 3), cols.31ff; Y. Maeyama, “Ancient stellar observations Timocharis, Aristyllus, Hipparchus, Ptolemy: the dates and accuracies”, Centaurus, 27 (1984), 280-310.

${ }^{27}$ G. Grasshoff, op. cit. (ref. 4)190-91.

${ }^{28}$ R. Nadal and J.-P. Brunet, Archive for history of exact sciences, xxix(1984), 201-36 and $\mathrm{xl}(1989), 305-54$.

${ }^{29}$ K. Pickering. DIO 9.1 (1999), 26-29.

${ }^{30}$ D. Rawlins, DIO 1.1 (1991), 62-63.

${ }^{31}$ O. Neugebauer, A history of ancient mathematical astronomy, (3 vols., Berlin, 1975), 281.

32 J. Meeus, Astronomical algorithms, $2^{\text {nd }}$ edition (Richmond, 1998), 93. 
${ }^{33}$ The formulae here are also in G. Grasshoff, op. cit. (ref. 4), 177-78. However, there is a misprint in Grasshoff's Eq. (5.7), and in general he omits the details on the signs needed to properly deal with the inverse tangent.

${ }^{34}$ K. Pickering, op. cit. (ref. 29). 